\title{
Patching versus weekend atropine for treatment of moderate to severe childhood amblyopia in Fife, Scotland
}

\author{
V. Swetha E. Jeganathan ${ }^{1,2 *}$, Shona Sutherland ${ }^{3}$, Jennifer Skillen $^{3}$, Penny Shackleton ${ }^{3}$, Debbie Wilson $^{3}$, Gilian Mo $^{3}$ and Andrew Blaikie ${ }^{3}$ \\ ${ }^{1}$ Department of Ophthalmology, University Hospital, Ayr, Scotland, United Kingdom \\ ${ }^{2}$ University of Edinburgh, Scotland, United Kingdom \\ ${ }^{3}$ Department of Ophthalmology, NHS Fife, Scotland, United Kingdom
}

\begin{abstract}
Objective: Patching has been the mainstay of amblyopia treatment. The Pediatric Eye Disease Investigation Group's (PEDIG) recent clinical trials have revolutionized our approach to amblyopia treatment and highlighted the effectiveness of atropine as a means to 'penalize' the good eye in amblyopia children. However, to date no published comparative studies in the UK have examined the effectiveness of amblyopia treatment per PEDIG recommendations. Our prospective study aimed to evaluate the efficacy of penalization with patching versus 'weekend' atropine for the treatment of moderate to severe amblyopia in children, served by NHS Fife hospital eye service.

Methods: Amblyopic children (3 to 7 years old) with 20/40 to 20/200 visual acuity (VA) were recruited from January 2011 to December 2012. Children either received patch occlusion or 'weekend' atropine penalization based on parental preference after reading an informational document on penalization options and discussing the information with an orthoptist and/or ophthalmologist. Parents and caregivers were then offered their first-choice treatment option. Children who initially received patch therapy but showed poor improvement or compliance were shifted to atropine penalization and vice versa. Success was defined as an increase of 2 or more lines of VA.

Results: We examined 158 children (58\% males, 42\% females). The cause of the amblyopia was squint (73\%), anisometropia (47\%) and 'mixed' cause (20\%). Forty percent of the children received 'weekend' atropine and $60 \%$ patching, with an overall success rate of $88 \%$ and $49 \%$, respectively. Amongst those patched, $7 \%$ showed poor compliance and $4 \%$ had limited improvement; they were switched to atropine. Although the final mean VA (0.3 logMAR) was similar between the two modalities, improvement was faster in the 'pure' atropine group (average treatment period of 14.5 weeks versus 26 weeks for patching). Reverse amblyopia occurred in two cases with atropine and optical penalization, but was reversible upon cessation of therapy.

Conclusions: Our study demonstrated that intense and extended regimens are not initially needed in amblyopia therapy, concurring with PEDIG findings. 'Weekend' atropine was more effective than patching due to superior patient compliance and treatment response. However, reverse amblyopia occurred with concurrent optical penalization, warranting more vigilant monitoring in this treatment group.
\end{abstract}

\section{Background}

Amblyopia, a disorder of reduced visual function from abnormal visual experience caused by strabismus, anisometropia or visual form deprivation, $[1,2]$ is the most common cause of monocular visual impairment in children and young and middle-aged adults, affecting $2 \%$ to $5 \%$ of the general population [3]. Early diagnosis and identification are important as the condition is usually reversible if treated within the critical period for visual development [4]. Amblyopia is more responsive to treatment among children younger than seven [5]. Individuals with amblyopia experience an increased lifetime risk of vision loss, with almost three times the risk of visual impairment in their better-seeing eye to less than 20/40 ( $\log$ MAR 0.3) compared to people without amblyopia [6]. Long-term consequences include increased risk of bilateral blindness [7].

Amblyopia treatment is more cost-effective than other interventions because the VA benefit occurs at a young age [8]. Fewer amblyopes age 49+ years completed higher university degrees [9]. Amblyopia treatment resulted in 2053 to 2509 \$/QALY gained [10]. Treatment improves vision in the amblyopic eye and reduces the danger of blindness in the other eye while aiding fusion, thereby facilitating maintenance of eye alignment and potentially improving stereoscopic appreciation, making individuals less accident prone [2]. Treatment also creates a better sighted "spare tire" should trauma or disease claim the sound eye. Screening and treatment for children with amblyopia can reduce the well-reported burden of future visual disability when left untreated.

\section{Treatment options}

Patching and atropine penalization, the primary amblyopia treatments, are effective in reducing the overall prevalence and severity of visual loss in this population [3]. For more than 250 years, extended patching of the unaffected eye to ensure the use of the affected eye was advocated for amblyopia treatment [1]. Ad hoc patching has

Correspondence to: Dr. V. Swetha E. Jeganathan, Department of Ophthalmology, University Hospital Ayr, Dalmellington Road, Ayr, South Ayrshire KA9 6DX Scotland, United Kingdom; E-mail: vswetha@ausdoctors.net

Key words: amblyopia, atropine penalization, visual acuity, patch therapy, occlusion therapy

Received: March 25, 2016; Accepted: April 18, 2016; Published: April 22, 2016 
been associated with poor compliance, social stigma and regimes vary greatly by individual clinicians with different prescribing traits [11]. Atropine penalization can be varied daily/alternate day, weekend, with or without optical penalization [12]. To date, research on the best treatment practice for amblyopia in Scotland is limited.

A series of recent randomized controlled treatment trials from the PEDIG have provided a more evidence-based approach to treatment, offering novel evidence that lesser amounts of patching are as effective as more intense regimens and that atropine penalization is often as effective as patching [13-16]. Researchers demonstrated that weekendonly atropine use was as effective as daily use and 2 hours of patching improved moderate to severe amblyopia in children [17]. The PEDIG studies confirmed that the initial choice of treatment was not binding; if a patient did not respond satisfactorily, it was reasonable to prescribe more intense or different treatment $[13,18]$. Reduced lengths of regimen are also effective, with the potential maximum vision in an amblyopic eye being reached in 3 to 4 months with treatment compliance. Prior to these PEDIG studies, children's treatment lasted 12 months or more before intervention success was deduced [17].

The PEDIG studies provide clinicians with more distinct guidelines with respect to the how and when of occlusion and penalization therapy of amblyopia. However, the evidence-based recommendations for amblyopia management have not widely translated into clinical practice changes [19]. The effect of recent evidence-based amblyopia research on current clinical practice in the UK has been sporadic and incomplete, with one-third of respondents indicating they made no changes whatsoever to their practice post-studies: Atropine was rarely recommended as a first- line treatment, with occlusion generally considered more effective [20].

Using lessons learned from the PEDIG studies, we designed a clinical protocol to evaluate the effectiveness of reduced patching versus 'weekend' atropine penalization to assess whether the treatment regime would be effective even with severe amblyopia. We aimed to tailor the therapy to achieve the best possible compliance and visual outcome with the least negative impact on the patient and family. We sought to improve service delivery by studying the time required for improvement so that we could better understand if the review process was effective or required alterations. To date, no such comparative studies on the effectiveness of amblyopia treatment have been published in the UK.

\section{Materials and methods}

We performed a prospective observational study of children, 3 to 7 years old, treated for moderate to severe amblyopia at NHS Fife between January 2011 and December 2012. The eligibility criteria included VA in the amblyopic eye between 20/40 (logMAR 0.3 ) and 20/200 (logMAR 1.0), intereye acuity difference of 3 or more logMAR lines, differences in refractive error between eyes of more than 1 dioptre for hyperopia and 1.5 dioptre for astigmatism, and the presence or history of an amblyogenic factor for strabismus and/or anisometropia. These children used optical correction (if required) for at least 4 months prior to our study and were available for at least 6 months of followup. Children who had a poor fixation pattern in the amblyopic eye or starting VA less than 20/200 (1.0 logMAR) were excluded.

Parents/caregivers were offered the option of weekend atropine or patching. Optical penalization was additionally available at the discretion of the supervising consultant. Parents received an information sheet on the subject's treatment details, including aims, importance of compliance and the follow-up as well as potential complications (e.g., possibility of reversal). All concerns were fully addressed and explained before the start of the treatment. Informed consent was obtained. Parents were offered the first choice of treatment option based on their preference and encouraged close work during treatment. Patient details were entered into the departmental database on the same day as treatment initiation.

\section{Treatment protocol}

Subjects' baseline measurements included logMAR VA, strabismus examination, cycloplegic refraction and ophthalmoscopy of the fundus. The VA in the amblyopic eye was the primary efficacy outcome measure. Refractive error was measured using cycloplegic retinoscopy 30 minutes after topical administration of $1 \%$ cyclopentolate.

Children with 'weekend' atropine had $1 \%$ atropine sulphate for a weekend or on 2 consecutive days during the week, with a 5-day gap between instillation of drops. Optical penalization was added only if subjects showed no improvement after 3 months. Those who chose patching had 2- hour patching twice a week initially, increasing to 6 or more hours daily if the VA worsened. Children who received patching but showed poor improvement or compliance were shifted to atropine penalization and vice versa.

Follow-up occurred every 4 to 6 weeks following treatment, lasting up to 6 months if the VA continued to improve. Extra visits were conducted at the clinicians' discretion. During each visit, the clinician rechecked the VA and determined the mean of each VA following Holladay and Prager [21]. Children with squints were monitored for changes in their angles with treatment and clearly noted fixation preference up close and at a distance. All subjects were evaluated for possible side-effects and compliance to the treatment protocol. Parents kept a treatment diary which was reviewed at each follow-up visit.

We maintained a flexible clinical approach regarding treatment modality switches, with decisions made based on treatment response, compliance and complications. For instance, atropine was discontinued if a side-effect was reported or the VA from the sound eye dropped by an equal amount or more than $2 \log M A R$ lines. Patients who did not attend a follow-up visit were contacted and offered another appointment no later than 3 weeks. If patients could not be contacted, a standard letter was sent out strongly advising them to stop amblyopia treatment unless clinically supervised. Finally, treatment was discontinued if no further improvement in VA was noted at follow-up visits. The endpoint of our study was when treatment was ceased. Successful treatment was defined as 2 or more lines of improvement from baseline VA or VA of $20 / 30(\log$ MAR 0.2$)$ or better in the amblyopic eye.

\section{Statistical methods}

The rate of successful treatment and final mean of VA of the three groups were calculated using SPSS software, version 16 (Chicago, IL, USA). Differences between treatment groups were evaluated using a Fischer exact test; differences in continuous outcomes between treatment groups were analyzed using an independent sample $t$-test. All reported $p$-values are 2-tailed.

\section{Results}

We collected data from 158 children (58\% males, $42 \%$ females), 3 to 7 years old (mean age 5 years). The cause of the amblyopia was squint (73\%), anisometropia (47\%) and 'mixed' cause (20\%). Forty per cent of subjects received 'weekend' atropine penalization; $60 \%$ chose 
patching initially, although $7 \%$ had poor compliance and $4 \%$ showed limited improvement, resulting in them being switched to 'weekend' atropine (Figure 1). No subjects in the atropine penalisation group were switched to patching.

In Figure 2, the mean improvement of VA in the amblyopic eye at 6 months was greater in the atropine compared to patching $(\mathrm{p}<0.01)$.

Table 1 shows the mean VA in the amblyopic eye before and after treatment in the 3 subgroups: pure atropine, patching (poor compliance) and patching (limited response). The mean final VA was approximately $0.3 \operatorname{logMAR}$ in all 3 groups. The magnitude of VA improvement was greatest in the initial patching group that showed limited response and was switched to 'weekend' atropine (4 lines of VA gain). However, improvement was fastest in the atropine only group (14.5 weeks, compared to 26 weeks in patching), with 3 lines of VA improvement. The relative treatment effect did not vary according to age, depth or cause of amblyopia. Following treatment, the acuity of the amblyopic eye averaged approximately 2 lines worse than the sound eye.

Severe amblyopes $(\log M A R>0.6)$ did poorly with patching; all were switched to atropine. The outcomes of severe amblyopes were quite impressive with 'weekend' atropine (6.1 lines of improvement at the end of follow-up). Those with VA $0.825 \log$ MAR in the amblyopic eye achieved VA of $0.3 \log$ MAR on the third visit. These results were better when only squints were present. No cases of pure anisometropia occurred within the severe group, and poorer outcomes were noted in 2 cases of severe amblyopia with combined amblyogenic factors.

The mean overall success rate of atropine penalization treatment-

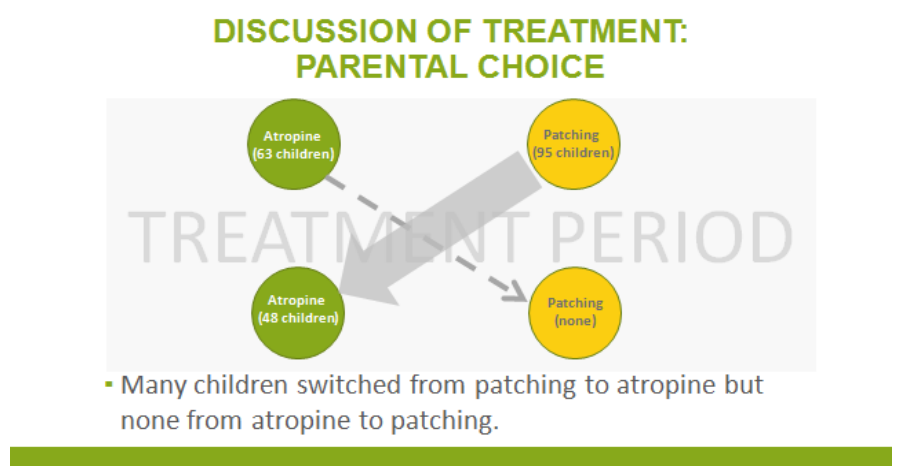

Figure 1. How subjects were included in the study.

\section{MEAN CHANGE IN LOGMAR VA OF THE AMBLYOPIC EYE WITH TREATMENT}

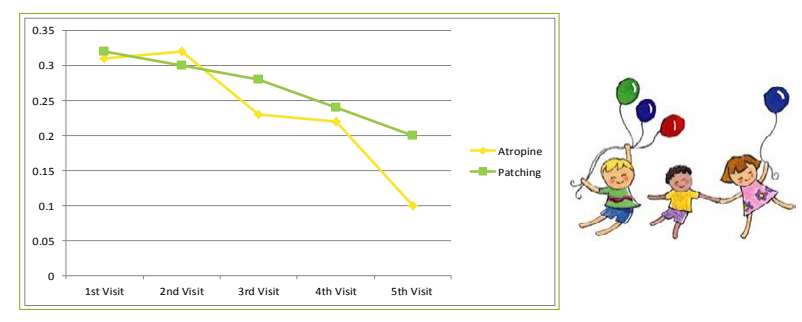

The mean improvement of VA in the amblyopic eye at 6 months was greater in the atropine compared to patching ( $p<0.01)$.

Figure 2. Mean change in $\log$ MAR VA of the amblyopic eye with treatment.
Table 1. Mean VA before and after treatment in the 3 groups.

\begin{tabular}{|c|c|c|c|}
\hline & Pure atropine group & $\begin{array}{l}\text { Patch initially (poor } \\
\text { compliance; switched to } \\
\text { atropine) }\end{array}$ & $\begin{array}{l}\text { Patch initially } \\
\text { (limited } \\
\text { response; switched } \\
\text { to atropine) }\end{array}$ \\
\hline $\begin{array}{l}\text { Mean VA(before } \\
\text { treatment) }\end{array}$ & $0.60 \log \mathrm{MAR}(20 / 80)$ & $0.50 \log$ MAR $(20 / 63)$ & $\begin{array}{l}0.70 \text { logMAR } \\
(20 / 100)\end{array}$ \\
\hline $\begin{array}{l}\text { Mean VA(after } \\
\text { treatment) }\end{array}$ & $0.30 \log$ MAR $(20 / 40)$ & $0.40 \log$ MAR $(20 / 50)$ & $0.30 \log$ MAR $(20 / 40)$ \\
\hline $\begin{array}{l}\text { Mean length of } \\
\text { treatment (number } \\
\text { of weeks) }\end{array}$ & 14.5 weeks & $\begin{array}{l}4 \text { weeks patch therapy, } \\
22 \text { weeks atropine }\end{array}$ & $\begin{array}{l}6 \text { weeks patch therapy, } \\
20 \text { weeks atropine }\end{array}$ \\
\hline
\end{tabular}

Table 2. Summary of current studies on atropine penalization for amblyopia.

\begin{tabular}{|l|l|l|}
\hline & $\begin{array}{l}\text { Lines of VA } \\
\text { improvement }\end{array}$ & Treatment duration \\
\hline PEDIG (2009) [26] & 3.6 & 2 years \\
\hline PEDIG (2005) [27] & 2 & 17 months \\
\hline Repka (2005) [27] & 3.6 & 2 years \\
\hline PEDIG (2002) [28] & 2.84 & 6 months \\
\hline Foley-Nolan (1997) [29] & 6 & 7 months \\
\hline Repka and Ray (1993) [30] & 2 & 1 year and 1 month \\
\hline
\end{tabular}

achieving VA 20/30 (logMAR 0.2) or better at the end of follow-upwas $88 \%$ compared to $49 \%$ with patching. Atropine had a slightly higher degree of acceptability, which was attributed to good treatment compliance and fewer side effects. Two cases of amblyopia reversals with atropine and optical penalization were exacerbated by missed appointments, but the effects were transient and resolved completely when the atropine drops and plano glasses were stopped.

\section{Discussion}

Our study compared the consequences of weekend atropine penalization and patching in managing moderate to severe amblyopia in children ( 3 to 7 years old). We used an initial dose of either 2 hours patching daily or twice weekly atropine, resulting in a success rate of $49 \%$ and $88 \%$, respectively. Atropine success rates have been quoted as ranging from $78 \%$ to $100 \%$, varying across weekend and daily dosage [22-25]. Pharmacologic penalization with atropine has been shown to be more effective than patching therapy and to have a higher level of patient acceptability $[1,3,12]$. In our study, VA improvement was 3 lines at the 6-month follow-up, consistent with other published studies, where an improvement of 2 to 6 lines occurred following atropine treatment (Table 2).

Atropine's success might stem from its superior compliance, which is ensured upon instillation [12,29,31] .Side effects (e.g., facial flushing, fever, irritation, light sensitivity, allergy) were rare in our study. Using atropine twice weekly blurs the normal eye at near fixation (preventing accommodation and near vision), but allows for peripheral binocularity. Atropine use results in the selective degradation of high spatial frequencies within the retinal image, which may be critical in amblyopia treatment [32]. One atropine drop affects accommodation for 8 days [33], although near vision returns to 20/20 by day 5 . In our study, the average response to atropine penalization was quicker (14.5 weeks versus 26 weeks for patching), and no time was wasted switching to different modalities. These findings might affect how prescribed atropine penalization is utilized to maximize effectiveness while minimizing impact on school performance [33]. 
In the PEDIG studies, only 1 of 372 patients had reverse amblyopia [26]. In our study, two patients developed reverse amblyopia with atropine and optical penalization that were exacerbated by missed appointments. However, the effects were transient and resolved completely when the atropine drops and plano glasses were stopped. Reversals are typically of low incidence and largely transient if treatment is promptly discontinued. The incidence of reverse amblyopia is higher with combined optical and atropine penalization treatment as its effects might be synergistic [34], highlighting the need for strict careful patient monitoring of a fixation switch or drop in the sound eye. As a precaution, we currently provide only a 4-week supply of atropine and emphasize that treatment must stop at 4 weeks, regardless. Parents must understand the importance of being seen by the deadline. The combined optical and atropine penalization treatment system advises stopping treatment after 2 visits if no improvement is evident [35].

We found only a $49 \%$ success rate with patching. Of those patched, $7 \%$ had poor compliance and $4 \%$ showed limited treatment response. Success rates between $30 \%$ and $95 \%$ have been reported for patching [22,36-38]. The complete success of patching therapy is dependent on the patient's compliance. Noncompliance is higher as the child can easily remove the patch. Poor compliance to patching might stem from its obtrusive nature, disruption of binocularity, poor cosmetic appearance and psychological or social stigma. Although patching offers immediate reversibility and absence of systemic side effects, local skin irritation and allergy can occur with adhesive patch use. Parents can become frustrated by the constant negotiating with the child. A self- reported compliance with eye patching revealed that only $54 \%$ of parents achieved patching recommendations [39], and perceived self-efficacy was positively associated with compliance while perceived prohibition of the child's activities was negatively associated with compliance [39].

Studies have shown 'weekend' atropine to be as effective as patching for children with severe amblyopia, encouraging its role as first-line treatment for amblyopia $[1,18,40]$. Atropine penalization might be useful for severe amblyopes, especially recurrences, who are unwilling to wear patches $[41,42]$. The PEDIG treatment of severe amblyopia with 'weekend' atropine found 5.1 lines improvement with atropine penalization (compared to 6.1 lines in our study) [18].

We followed our cohort for at least 6 months. Follow-up regimes in the literature varied from 13 to 31.2 weeks for atropine treatment [22,43-45]. The PEDIG weekend atropine paper reported follow- up at 5 weeks, 16 weeks and 6 months $[14,16,46]$. The Lancet 2006 panel review recommended monitoring VA every 6 to 12 weeks whilst on weekend atropine [47]. Our study established that children can achieve maximum vision in their amblyopic eye within 4 to 6 months of treatment; thus, treatment can be stopped earlier in certain cases. A ceiling effect on the rate of improvement of the amblyopic eye likely occurs, at which point additional atropine drops or patching does not result in further improvement. Thus, overall treatment can be completed within a relatively short period of time. Nevertheless, minor variation exists contingent on compliance.

\section{Limitations}

Selection bias in our study might have occurred given its openlabel design. Clinician bias might exist from tried and tested patching. Clinicians had to resist combining treatments to allow the effects to build rather than swapping/discontinuing treatment in early stages. In the PEDIG study, treatment was changed only if no or limited improvement occurred at 16 weeks [13]. Individual variability might occur in response to treatment, especially patching without continuous electronic compliance monitoring. Our 6-month outcomes cannot provide information on the ultimate outcome of our amblyopia treatment. Deterioration in VA has been reported in longterm retrospective studies $[48,49]$. Regression can occur following the cessation of treatment, thereby reducing the lifetime benefit of therapy. As most patients in our study had only moderate amblyopia, we caution against extrapolating these findings to patients with more severe amblyopia. Moreover, the outcome assessments were unmasked and might limit the findings' validity. We did not assess the impact of amblyopia on quality of life or school performance.

\section{Future directions}

Established frameworks for knowledge translation, such as the Knowledge-to-Action Cycle, can help implement new knowledge gleaned from the PEDIG studies into actual clinical practice [50]. Many questions remain for the next generation of PEDIG amblyopia studies: when to abandon the initial treatment, what subsequent treatment should be and how to wean treatment when the maximum response has been achieved. Further studies should examine why some children are resistant to treatment whereas others seem to respond quickly and completely. As amblyopia is a neural disorder that results from abnormal stimulation of the brain during critical periods of visual development, it is essential to establish the neural mechanisms of amblyopia in order to devise better treatment strategies. A more standardized assessment of immediate and long-term effects of amblyopia and its treatment on the health-related quality of life is warranted.

\section{Conclusions}

Intense and extended regimens are not initially needed in amblyopia therapy. Although the outcome in both of our treatment modalities was similar, 'weekend' atropine penalization had superior patient acceptance, compliance and a faster treatment responsefurther asserting its role as a first line treatment for amblyopia. Patients and their parents should be educated about the need for regular followup and the risk of potential vision loss from reverse amblyopia. We will continue to examine our study cohort until the ages of 10 to 15 to determine their long-term VA and monitor for regression.

\section{References}

1. Stewart CE, Moseley MJ, Fielder AR (2011) Amblyopia therapy: an update. Strabismus 19: 91-98. [Crossref]

2. Grant S, Moseley MJ (2011) Amblyopia and real-world visuomotor tasks. Strabismus 19: 119-128. [Crossref]

3. Webber AL (2007) Amblyopia treatment: an evidence-based approach to maximising treatment outcome. Clin Exp Optom 90: 250-257. [Crossref]

4. Morishita H, Hensch TK (2008) Critical period revisited: impact on vision. Curr Opin Neurobiol 18: 101-107. [Crossref]

5. Holmes JM, Lazar EL, Melia BM, Astle WF, Dagi LR, et al. (2011) Effect of age on response to amblyopia treatment in children. Arch Ophthalmol 129: 1451-1457. [Crossref]

6. Nilsso J (2007) The negative impact of amblyopia from a population perspective: untreated amblyopia almost doubles the lifetime risk of bilateral visual impairment. $\mathrm{Br}$ J Ophthalmol 91: 1417-1418. [Crossref]

7. van Leeuwen R, Eijkemans MJ, Vingerling JR, Hofman A, de Jong PT, et al. (2007) Risk of bilateral visual impairment in individuals with amblyopia: the Rotterdam study. Br J Ophthalmol 91: 1450-1451. [Crossref]

8. König HH, Barry JC (2004) Cost effectiveness of treatment for amblyopia: an analysis based on a probabilistic Markov model. Br J Ophthalmol 88: 606-612. [Crossref] 
9. Chua B, Mitchell P (2004) Consequences of amblyopia on education, occupation, and long term vision loss. Br J Ophthalmol 88: 1119-1121. [Crossref]

10. Membreno JH, Brown MM, Brown GC, Sharma S, Beauchamp GR (2002) A costutility analysis of therapy for amblyopia. Ophthalmology 109: 2265-2271. [Crossref]

11. Kanonidou E (2011) Amblyopia: a mini review of the literature. Int Ophthalmol 31: 249-256. [Crossref]

12. Li T, Shotton K (2009) Conventional occlusion versus pharmacologic penalization for amblyopia. Cochrane Database Syst Rev : CD006460. [Crossref]

13. Gunton KB (2013) Advances in amblyopia: what have we learned from PEDIG trials? Pediatrics 131: 540-547. [Crossref]

14. Wallace DK, Kraker RT, Beck RW, Cotter SA, et al. (2011) Randomized trial to evaluate combined patching and atropine for residual amblyopia. Arch Ophthalmol 129: 960-962. [Crossref]

15. Rees MG, Woo CL, Optom B (2007) Pediatric eye disease investigator group amblyopia treatment review. Am Orthopt J 57: 99-103. [Crossref]

16. Kowal L (2002) PEDIG study on amblyopia; vision therapy by atropine penalization versus occlusion. Binocul Vis Strabismus Q 17: 275. [Crossref]

17. Repka MX, Holmes JM (2012) Lessons from the amblyopia treatment studies. Ophthalmology 119: 657-658. [Crossref]

18. Repka MX, Kraker RT, Beck RW, Birch E, Cotter SA, et al. (2009) Treatment of severe amblyopia with weekend atropine: results from 2 randomized clinical trials. $J$ AAPOS 13: 258-263. [Crossref]

19. Fleck BW (2003) Amblyopia therapy. Br J Ophthalmol 87: 255-256. [Crossref]

20. Newsham D (2010) The effect of recent amblyopia research on current practice in the UK. Br J Ophthalmol 94: 1352-1357. [Crossref]

21. Holladay JT, Prager TC (1991) Mean visual acuity. Am J Ophthalmol 111: 372-374. [Crossref]

22. Medghalchi AR, Dalili S (2011) A Randomized Trial of Atropine vs Patching for Treatment of Moderate Amblyopia. Iran Red Crescent Med J 13: 578-581. [Crossref]

23. Ron A, Nawratzki I (1982) Penalization treatment of amblyopia: a follow-up study of two years in older children. J Pediatr Ophthalmol Strabismus 19: 137-139. [Crossref]

24. Doshi NR, Rodriguez ML (2007) Amblyopia. Am Fam Physician 75: 361-367. [Crossref]

25. Granet DB, Christian W, Gomi CE, Banuelos L, Castro E (2006) Treatment options for anisohyperopia. J Pediatr Ophthalmol Strabismus 43: 207-211. [Crossref]

26. Pediatric Eye Disease Investigator Group (2009) Pharmacological plus optica penalization treatment for amblyopia: results of a randomized trial. Arch Ophthalmol 127: 22-30. [Crossref]

27. Repka MX, Wallace DK, Beck RW, Kraker RT, Birch EE, et al. (2005) Two-year follow-up of a 6-month randomized trial of atropine vs patching for treatment of moderate amblyopia in children. Arch Ophthalmol 123: 149-157. [Crossref]

28. Pediatric Eye Disease Investigator Group. (2002) A randomized trial of atropine vs. patching for treatment of moderate amblyopia in children. Arch Ophthalmol 120: 268278. [Crossref]

29. Foley-Nolan A, McCann A, O'Keefe M (1997) Atropine penalisation versus occlusion as the primary treatment for amblyopia. Br J Ophthalmol 81: 54-57. [Crossref]

30. Repka MX, Ray JM (1993) The efficacy of optical and pharmacological penalization. Ophthalmology 100: 769-774. [Crossref]

31. Suttle CM (2010) Active treatments for amblyopia: a review of the methods and evidence base. Clin Exp Optom 93: 287-299. [Crossref]
32. Kulp MT, Foster NC, Holmes JM, Kraker RT, Melia BM, et al. (2012) Effect of ocular alignment on emmetropization in children $<10$ years with amblyopia. Am J Ophthalmol 154: 297-302. [Crossref]

33. Matta N, Silbert DI (2013) Effects of atropine on the accommodative system: parameters to consider in its use for penalization treatment of amblyopia. Binocul Vis Strabolog Q Simms Romano 28: 39-44. [Crossref]

34. Morrison DG, Palmer NJ, Sinatra RB, Donahue S (2005) Severe amblyopia of the sound eye resulting from atropine therapy combined with optical penalization. $J$ Pediatr Ophthalmol Strabismus 42: 52-53. [Crossref]

35. Kaye SB, Chen SI, Price G, Kaye LC, Noonan C, et al. (2002) Combined optical and atropine penalization for the treatment of strabismic and anisometropic amblyopia. $J$ AAPOS 6: 289-293. [Crossref]

36. Scott WE, Kutschke PJ, Keech RV, Pfeifer WL, Nichols B, et al. (2005) Amblyopia treatment outcomes. J AAPOS 9: 107-111. [Crossref]

37. JT Flynn, G Woodruff, JR Thompson, F Hiscox, W Feuer, et al. (1999) The therapy of amblyopia: an analysis comparing the results of amblyopia therapy utilizing two pooled data sets. Trans Am Ophthalmol Soc 97: 373-90. [Crossref]

38. Flynn JT, Schiffman J, Feuer W, Corona A (1998) The therapy of amblyopia: an analysis of the results of amblyopia therapy utilizing the pooled data of published studies. Trans Am Ophthalmol Soc 96: 431-450. [Crossref]

39. Searle A, Norman P, Harrad R, Vedhara K (2002) Psychosocial and clinical determinants of compliance with occlusion therapy for amblyopic children. Eye (Lond) 16: 150-155. [Crossref]

40. Repka MX, Kraker RT, Beck RW, Birch E, Cotter SA, et al. (2009) Treatment of severe amblyopia with atropine: Results from 2 randomized clinical trials. J AAPOS 13: 529. [Crossref]

41. Holmes JM, Repka MX, Kraker RT, Clarke MP (2006) The treatment of amblyopia Strabismus 14: 37-42.

42. Lempert $P$ (2009) Treatment of severe amblyopia with weekend atropine: Results from 2 randomized clinical trials. J AAPOS 13: 528-529. [Crossref]

43. Travi GM, Schnall BM, Lehman SS, Kelly CJ, Hug D, et al. (2005) Visual outcome and success of amblyopia treatment in unilateral small posterior lens opacities and lenticonus initially treated nonsurgically. J AAPOS 9: 449-454. [Crossref]

44. Repka MX, Kraker RT, Beck RW, Holmes JM, Cotter SA, et al. (2008) A randomized trial of atropine vs patching for treatment of moderate amblyopia: follow-up at age 10 years. Arch Ophthalmol 126: 1039-44. [Crossref]

45. Leone J, Georgievski Z, Koklanis K (2010) Atropine treatment of amblyopia: is a swap in fixation necessary? J Pediatr Ophthalmol Strabismus 47: 270-276. [Crossref]

46. Kushner BJ (2005) Concern about the Pediatric Eye Disease Investigator Group 2-year follow-up study. Arch Ophthalmol 123: 1615-1616. [Crossref]

47. Holmes JM, Clarke MP (2006) Amblyopia. Lancet 367: 1343-1351. [Crossref]

48. Levartovsky S, Oliver M, Gottesman N, Shimshoni M (1995) Factors affecting long term results of successfully treated amblyopia: initial visual acuity and type of amblyopia. Br J Ophthalmol 79: 225-228. [Crossref]

49. Levartovsky S, Oliver M, Gottesman N, Shimshoni M (1998) Long-term effect of hypermetropic anisometropia on the visual acuity of treated amblyopic eyes. $\mathrm{Br} J$ Ophthalmol 82: 55-58.

50. Jin YP, Chow AH, Colpa L, Wong AM (2013) Clinical translation of recommendation from randomized clinical trials on patching regimen for amblyopia. Ophthalmology 120: 657-662. [Crossref]

Copyright: (C2016 Jeganathan VSE. This is an open-access article distributed under the terms of the Creative Commons Attribution License, which permits unrestricted use, distribution, and reproduction in any medium, provided the original author and source are credited. 